\title{
openheart Does valvuloarterial impedance impact prognosis after surgery for severe aortic stenosis in the elderly?
}

\author{
Minako Katayama, ${ }^{1}$ Mohammad Q Najib, ${ }^{1}$ Punnaiah C Marella, ${ }^{1}$
} M'hamed H Temkit, ${ }^{2}$ Marek Belohlavek, ${ }^{1}$ Hari P Chaliki ${ }^{1}$

To cite: Katayama $\mathrm{M}$, Najib MQ, Marella PC, et al. Does valvuloarterial impedance impact prognosis after surgery for severe aortic stenosis in the elderly?. Open Heart 2015;2:e000241. doi:10.1136/openhrt-2015000241

Received 16 January 2015 Revised 26 May 2015 Accepted 2 June 2015
CrossMark

\author{
${ }^{1}$ Division of Cardiovascular \\ Diseases, Mayo Clinic, \\ Scottsdale, Arizona, USA \\ ${ }^{2}$ Division of Health Sciences \\ Research, Mayo Clinic, \\ Scottsdale, Arizona, USA
}

Correspondence to Hari P Chaliki; chaliki.hari@mayo.edu

\section{ABSTRACT}

Background: Valvuloarterial impedance (Zva) was introduced as a prognostic measure in patients with aortic stenosis (AS). However, it is unclear whether Zva has a prognostic impact on survival after surgical aortic valve replacement (AVR) in patients with severe AS with preserved ejection fraction (EF).

Methods: We retrospectively reviewed 929 consecutive patients who had AVR. We investigated 170 elderly patients (age $>65$ years, mean 76 years) who had AVR secondary to severe AS (mean gradient $\geq 40 \mathrm{~mm} \mathrm{Hg}$; aortic valve area $\leq 1 \mathrm{~cm}^{2}$; peak velocity $\geq 4 \mathrm{~m} / \mathrm{s}$ ). Patients with $\mathrm{EF}<50 \%$, greater than moderate aortic regurgitation, prior heart surgery and concomitant mitral or tricuspid valve surgery were excluded. Zva was calculated and the patients were divided into two groups; low Zva, Zva $<4.3 \quad(n=82)$ and high Zva, Zva $\geq 4.3 \quad(n=88)$. The end point was allcause of death. Survival curves were calculated according to Kaplan-Meier method.

Results: Age, prevalence of hypertension, diabetes, chronic kidney disease (CKD), atrial fibrillation, symptoms, EF, E/e' and concomitant coronary artery bypass graft were not different between the groups. Survival was not different between the groups at 5 years $(70 \%$ in low Zva and $81 \%$ in high $Z v a ; p=0.21)$ and for the entire follow-up period $(p=0.23)$. Only age was a significant factor in predicting survival by multivariate analyses in Cox proportional hazards model after adjusting for Zva, CKD, atrial fibrillation and hypertension.

Conclusions: Our results suggest that preoperative Zva does not have a prognostic impact on postoperative survival in elderly patients with severe AS with preserved EF. Further investigation is needed to elucidate the controversial results.

\section{INTRODUCTION}

In the elderly, aetiologies of aortic stenosis (AS) are degeneration and calcification of the valve, which are closely related to atherosclerosis and other comorbidities such as hypertension, hypercholesterolaemia, coronary artery disease and chronic kidney disease. ${ }^{1}$ A stenotic aortic valve is the biggest

\section{KEY MESSAGES}

What is already known about this subject?

- In elderly patients with aortic stenosis (AS), the myocardium of the left ventricle (LV) is exposed to stress not only by the stenotic aortic valve but also by systemic arterial resistance. Valvuloarterial impedance (Zva), which represents both valvular load and arterial load, has been introduced as a good measure for risk stratification and prognostic assessment in AS.

What does this study add?

- Given continued excess of afterload, higher Zva is expected to retain its impact on survival after surgical aortic valve replacement (AVR). However, in our study, there was no significant difference in postoperative survival between patients with high Zva versus low Zva after AVR. The Zva parameter needs more attention and further investigation to reconcile these controversial results.

How might this impact on clinical practice?

- Although Zva is an additional measure of global haemodynamic load, careful interpretation and application of Zva are essential in clinical practice.

factor in increased left ventricular (LV) afterload, but afterload due to systemic resistance such as hypertension is also significant. ${ }^{2}$

Recent studies emphasised the importance of a new concept called 'valvulo-arterial impedance (Zva)', which not only takes the valvular load into account but also does so for the arterial load in the assessment of $\mathrm{AS}^{2-4}$ It was suggested that Zva is superior to standard measures of AS severity. ${ }^{4}$ More importantly, several reports support the significance of Zva as a good prognostic measure $^{3-7}$ in patients with AS.

However, it is unclear whether Zva retains its prognostic impact on survival after conventional aortic valve replacement (AVR), given continued excess of afterload in some patients. ${ }^{8}$ We hypothesised that patients with severe AS with preserved ejection fraction 
(EF) who have increased Zva have poorer prognosis even after successful AVR because of high-global haemodynamic load chronically causing LV myocardial structural damage and thus, the recovery of LV function and regression of LV hypertrophy is slower or irreversible.

The purpose of this study was to elucidate the prognostic significance of Zva for survival after surgical AVR.

\section{METHODS}

\section{Study group}

We retrospectively reviewed medical records of 929 consecutive patients of all ages who underwent surgical AVR between the years 1998 to 2011, at Mayo Clinic in Arizona. Among them, 752 patients were excluded due to the following exclusion criteria; age $\leq 64$ years old, less than severe AS, reduced LVEF $(\mathrm{EF}<50 \%)$, greater than moderate aortic regurgitation, prior valve surgery, concomitant mitral or tricuspid valve surgery. As a result, we selected 177 patients (age $>65$ years old) with AVR secondary to severe AS with preserved EF $(\geq 50 \%)$. The study was approved by the Mayo Foundation Institutional Review Board. Detailed baseline demographics including symptoms, comorbidities and echocardiographic variables, were recorded from the existing medical records documented at the time of preoperative assessment.

\section{Echocardiography}

Comprehensive echocardiographic examinations were performed before AVR using standardised methods. ${ }^{9-11}$ Blood pressure was measured by an arm-cuff sphygmomanometer at the time of echocardiography. LVEF was measured using biplane Simpson's method. Mitral valve inflow velocities by pulsed wave Doppler and septal mitral annulus velocity by tissue Doppler were measured and $\mathrm{E} / \mathrm{e}^{\prime}$ was calculated. The time velocity integral of $\mathrm{LV}$ outflow was measured by pulsed wave Doppler method from an apical long-axis view or five-chamber view, and LV outflow tract diameter was measured from a parasternal long-axis view for calculation of stroke volume (SV). SV was indexed by body surface area. Mean gradient and peak gradient of the aortic valve were measured by continuous wave Doppler using all possible windows to detect the optimal signal. Aortic valve area (AVA) was calculated by the continuity method. Severe AS was determined by Doppler echocardiography to be those who satisfy mean gradient $\geq 40 \mathrm{~mm} \mathrm{Hg}$, AVA $\leq 1 \mathrm{~cm}^{2}$ and peak velocity $\geq 4 \mathrm{~m} / \mathrm{s}$ as per the American College of Cardiology/American Heart Association guidelines. ${ }^{12}$

Zva was calculated according to the formula: ${ }^{23}$

$$
\begin{aligned}
\text { Zva }= & (\text { Systolic Blood Pressure } \\
& + \text { Mean Gradient of Aortic Valve }) \\
& \text { /Stroke Volume Index }
\end{aligned}
$$

Systolic blood pressure was measured at the time of echocardiography, and mean gradient of aortic valve and stroke volume index were obtained from the existing echocardiographic report.

Although Hachicha $e t a l^{4}$ presented prognostic significance of discrete ranges of $\mathrm{Zva}$ values (Zva $\leq 3.5$, $3.5<\mathrm{Zva}<4.5, \mathrm{Zva} \geq 4.5$ ), we chose two group comparison divided by median value of $\mathrm{Zva}$ in order to maintain adequate statistical power. Patients were divided into two groups based on Zva.

$$
\begin{aligned}
& \text { Low Zva } \quad \text { Zva }<4.3 \mathrm{~mm} \mathrm{Hg} / \mathrm{mL} \mathrm{m}^{2} \\
& \text { High Zva } \quad \text { Zva } \geq 4.3 \mathrm{~mm} \mathrm{Hg} / \mathrm{mL} \mathrm{m}^{2}
\end{aligned}
$$

Pulse pressure (PP) was measured as the difference between systolic and diastolic blood pressures. Systemic arterial compliance (SAC) was calculated according to the formula: ${ }^{2}$

$$
\mathrm{SAC}=\text { Stroke Volume Index }(\mathrm{SVI}) / \text { Pulse Pressure }(\mathrm{PP})
$$

\section{Follow-up}

The end point of this study was all-cause of death. The survival data were collected from medical records and the Social Security Death Index.

\section{Statistical analysis}

Before initiating the study, a power calculation was performed. The sample from our existing database included 177 patients with $77 \%$ survival at 5 years and $52 \%$ survival at 10 years. Power was approximated by considering a comparison of survival in participants with baseline Zva above the median level versus participants with baseline Zva no more than the medial level. A sample of 88 participants with Zva above the median and 89 participants with Zva below the median has $80 \%$ power $(\alpha 0.05)$ if survival at 5 years differs by at least $18 \%$ points $(68 \%$ vs $86 \%)$. This difference of $18 \%$ was estimated according to the study by Hachicha et al, which shows survival difference at 4 years of $23 \%$ between high Zva vs low Zva and 13\% between high Zva vs moderate Zva. ${ }^{4}$

Continuous variables were presented as mean \pm SD. Differences between groups were tested by unpaired $\mathrm{t}$ test in continuous variables and $\chi^{2}$ test or Fisher's exact test for categorical variables. Postoperative survival curves were calculated according to the Kaplan-Meier method and comparisons between groups were made with the log-rank test. The Cox proportional hazards model was used for univariate and multivariate analysis. Age, Zva, E/e', SAC, SVI and PP were incorporated into the model as continuous variables. Male gender, CKD, concomitant coronary artery bypass graft (CABG), atrial fibrillation and hypertension (HT) were incorporated as categorical variables. Variables that had $p$ value $<0.20$ in univariate analysis were incorporated into multivariate analysis. Statistical analyses were performed using JMP pro 10.0.0 (SAS Institute Inc, Cary, North Carolina, USA). A $p<0.05$ was considered statistically significant. 


\section{RESULTS}

Zva was calculated in 170 patients (7 patients were excluded due to missing data) and divided into two groups: low Zva (Zva $<4.3, \mathrm{n}=82)$; high $\mathrm{Zva}$ (Zva $\geq 4.3$, $\mathrm{n}=88$ ).

Patient characteristics and haemodynamic data were presented in table 1 . There were no significant differences in age, gender, prevalence of hypertension, diabetes, CKD (CKD, estimated glomerular filtration rate $<60 \mathrm{~mL} / \mathrm{min} / 1.73 \mathrm{~m}^{2}$ ), symptoms related to AS and atrial fibrillation,

$\mathrm{LVEF}, \mathrm{E} / \mathrm{e}^{\prime}$, and the rate of concomitant CABG at the time of AVR. Calculated AVA was significantly different between the groups and was smaller in the high Zva group as expected. Systolic blood pressure and mean gradient of aortic valve were significantly higher in the high Zva group, and stroke volume index (SVI) was significantly lower in the low Zva group, also as expected. Pulse pressure was higher and SAC was lower in the high Zva group.

We found the rate of death to be 25 out of $82(30 \%)$ in the low Zva group and 22 out of 88 (25\%) in the high Zva group. There was no significant difference in postoperative survival at 5 years $(70 \%$ in low $\mathrm{Zva}<4.3$, $81 \%$ in high $\mathrm{Zva} \geq 4.3, \mathrm{p}=0.21$ by log-rank test) and for the entire follow-up period $(\mathrm{p}=0.23$ by log-rank test; figure 1). Zva $\geq 4.3$ presented a HR for mortality of 0.70 (95\% CI 0.39 to $1.25, \mathrm{p}=0.23$ ) for the entire follow-up period.

Univariate and multivariate analysis in Cox proportional hazards model are presented in table 2. Multivariate analyses after adjusting for Zva, presence of CKD, atrial fibrillation and HT showed that only age

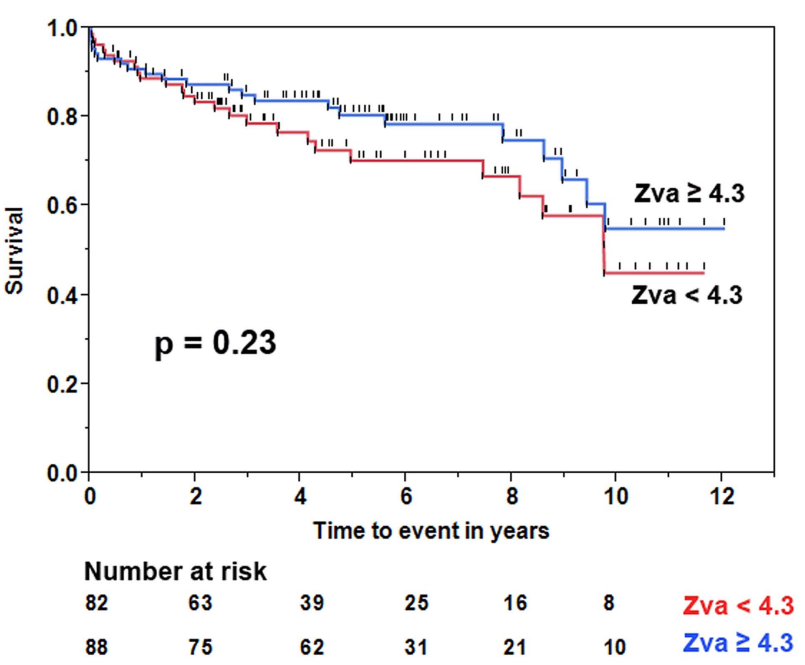

Figure 1 Postoperative survival curves. Survival curves of patients with Zva $<4.3$ and Zva $\geq 4.3$ are presented. No significant difference is observed between the groups $(p=0.23$ by log-rank test).

(HR 1.10 (95\% CI 1.04 to 1.17 ); $\mathrm{p}=0.0004$ ) was the significant factor in predicting survival after AVR.

\section{DISCUSSION}

We investigated the usefulness of Zva as a prognostic value in elderly patients with severe AS with preserved EF who underwent surgical AVR. A main finding of our study is that Zva does not have a prognostic impact on postoperative survival in patients with AS with preserved EF. Therefore, we had to reject our hypothesis. The significance of this study is that only elderly patients with

Table 1 Baseline characteristics and haemodynamic data

\begin{tabular}{|c|c|c|c|}
\hline & Low Zva <4.3 (n=82) & High Zva $\geq 4.3(n=88)$ & p Value \\
\hline Age & $77 \pm 6$ & $76 \pm 6$ & 0.8287 \\
\hline Male, $\%$ & $57 \%$ & $68 \%$ & 0.1427 \\
\hline $\mathrm{HT}, \%$ & $73 \%$ & $74 \%$ & 0.9185 \\
\hline $\mathrm{DM}, \%$ & $21 \%$ & $17 \%$ & 0.4882 \\
\hline CKD, \% & $35 \%$ & $34 \%$ & 0.8162 \\
\hline CABG, \% & $49 \%$ & $53 \%$ & 0.5463 \\
\hline Symptom, \% & $71 \%$ & $77 \%$ & 0.3306 \\
\hline Atrial fibrillation, \% & $5 \%$ & $8 \%$ & 0.5380 \\
\hline LVEF, \% & $66 \pm 6$ & $64 \pm 7$ & 0.1664 \\
\hline$E / e^{\prime}$ & $18 \pm 8$ & $19 \pm 10$ & 0.5141 \\
\hline AVA, $\mathrm{cm}^{2}$ & $0.78 \pm 0.14$ & $0.64 \pm 0.12$ & $<0.0001$ \\
\hline $\mathrm{BP}, \mathrm{mm} \mathrm{Hg}$ & $128 \pm 17$ & $142 \pm 18$ & $<0.0001$ \\
\hline $\mathrm{MG}, \mathrm{mm} \mathrm{Hg}$ & $54 \pm 13$ & $59 \pm 15$ & 0.0162 \\
\hline $\mathrm{SVI}, \mathrm{mL} / \mathrm{cm}^{2}$ & $51 \pm 7$ & $40 \pm 5$ & $<0.0001$ \\
\hline $\mathrm{BSA}, \mathrm{cm}^{2}$ & $1.88 \pm 0.24$ & $1.97 \pm 0.20$ & 0.0055 \\
\hline Zva, $\mathrm{mm} \mathrm{Hg} / \mathrm{mL} \mathrm{m}^{2}$ & $3.6 \pm 0.4$ & $5.1 \pm 0.7$ & $<0.0001$ \\
\hline Pulse pressure, $\mathrm{mm} \mathrm{Hg}$ & $58 \pm 14$ & $66 \pm 17$ & 0.0007 \\
\hline $\mathrm{SAC}, \mathrm{mL} / \mathrm{m}^{2} / \mathrm{mm} \mathrm{Hg}$ & $0.92 \pm 0.23$ & $0.63 \pm 0.16$ & $<0.0001$ \\
\hline
\end{tabular}


Table 2 Univariate and multivariate cox analysis for predictors of survival after AVR

\begin{tabular}{|c|c|c|c|c|}
\hline & \multicolumn{2}{|c|}{ Univariate analysis } & \multicolumn{2}{|c|}{ Multivariate analysis } \\
\hline & p Value & HR $(95 \% \mathrm{Cl})$ & p Value & HR (95\% Cl) \\
\hline Age & $<0.0001$ & $1.10(1.05$ to 1.15$)$ & 0.0004 & 1.10 (1.04 to 1.17$)$ \\
\hline Male gender & 0.2762 & $0.72(0.40$ to 1.30$)$ & & \\
\hline $\mathrm{Zva}\left(\mathrm{mm} \mathrm{Hg} / \mathrm{mL} \mathrm{m}^{2}\right)$ & 0.3268 & $0.85(0.62$ to 1.15$)$ & 0.4980 & 0.89 (0.63 to 1.23$)$ \\
\hline CKD & 0.1647 & 1.52 (0.83 to 2.73$)$ & 0.6293 & 1.17 (0.61 to 2.19$)$ \\
\hline CABG & 0.3198 & $1.33(0.75$ to 2.41$)$ & & \\
\hline Atrial fibrillation & 0.1061 & 2.35 (0.81 to 5.42$)$ & 0.2243 & 1.90 (0.63 to 4.60$)$ \\
\hline$E / e^{\prime}$ & 0.7662 & 0.99 (0.95 to 1.02$)$ & & \\
\hline HT & 0.1833 & $1.56(0.81$ to 2.24$)$ & 0.0864 & 0.79 (0.92 to 3.78$)$ \\
\hline $\mathrm{SAC}\left(\mathrm{mL} / \mathrm{m}^{2} / \mathrm{mm} \mathrm{Hg}\right)$ & 0.9961 & $0.99(0.27$ to 3.24$)$ & & \\
\hline $\mathrm{SVI}\left(\mathrm{mL} / \mathrm{cm}^{2}\right)$ & 0.8117 & $1.00(0.96$ to 1.03$)$ & & \\
\hline $\mathrm{PP}(\mathrm{mm} \mathrm{Hg})$ & 0.8437 & 0.99 (0.97 to 1.01$)$ & & \\
\hline
\end{tabular}

CABG, coronary artery bypass graft; CKD, Chronic Kidney Disease; HT, hypertension; SAC, systemic arterial compliance; SVI, stroke volume index; PP, pulse pressure.

severe high-gradient AS are targeted for prognostic observation after traditional surgical AVR.

Zva was introduced as a measure of global LV afterload in patients with $\mathrm{AS}^{2}{ }^{3}$ The systolic arterial pressure to SVI ratio, which is an approximation of arterial impedance, was employed in the formula. At the same time, decreased SVI due to excess concentric hypertrophy or decreased LV contraction, which are particularly observed in low-flow $\mathrm{AS}^{3}{ }^{3}$ is taken into consideration. That is to say, in addition to the valvular load imposed by AS, poor prognostic elements such as high-blood pressure, low arterial compliance and low SVI, are summed up in this formula. This composite measure of LV afterload, Zva, has shown prognostic significance in patients with AS in several reports. ${ }^{4-6}$ Results from our study are contradictory to these studies, likely due to a difference in the definition of primary end point, patient characteristics and length of follow-up.

Specifically, two studies by Rieck et $a t^{6}$ and Lancellotti et $a \tilde{l}$ showed that Zva was predictive of major cardiovascular events, that is, the combined end points of mostly aortic valve replacements and ischaemic cardiac events; Rieck et al showed poorer survival in high Zva group in mild-moderate AS (mean age 67 years) with less numbers of deaths, unlike our study, which enrolled patients with severe AS with AVR (mean age 76 years). A study by Hachicha et at had a mixed population of patients with moderate and severe AS, with a mean gradient ranging from $25 \pm 10 \mathrm{~mm} \mathrm{Hg}$ to $34 \pm 17 \mathrm{~mm} \mathrm{Hg}$, unlike our study with only severe AS patients, all of whom had a mean gradient of $\geq 40 \mathrm{~mm} \mathrm{Hg}$. Although 176 patients $(34 \%)$ who had AVR, among 522 patients, were presented as a subgroup, the details of haemodynamic data and patient characteristics of the subgroup were not shown. More importantly, most of the deaths, 78 out of 91 deaths $(86 \%)$, occurred in patients who were treated medically. It is not clear from the study whether some of the increased mortality in the higher Zva groups is related to denial of surgery despite symptomatic AS and/or comorbidities versus increased Zva.
In contrast to our study results, a recent study by Katsanos et $a l^{7}$ reported that Zva had an independent prognostic value in terms of overall mortality in 116 patients who underwent transcatheter aortic valve replacement (TAVR). However, higher occurrences of hypertension ( $49 \%$ vs $31 \% ; \mathrm{p}=0.06)$ and atrial fibrillation $(29 \%$ vs $14 \%$; $\mathrm{p}=0.06)$ in patients with elevated Zva were not adjusted in the multivariate analysis. More importantly, patients undergoing TAVR were typically older and likely with more comorbidities causing severe vascular burden when compared to our surgical AVR. This could explain the difference between the referenced and our study results.

Similar to our study, except for the EF criteria, Levy et al investigated the prognostic significance of Zva in 184 patients who had severe AS with decreased LVEF, and concluded that Zva did not have a prognostic role after AVR, ${ }^{8}$ which supports the results of our study, although the targeted patients had different characteristics. Another study by Jander et $a l^{13}$ reported that patients with 'low gradient' severe AS despite preserved EF, who likely have high Zva, ${ }^{3}{ }^{4}$ have a prognosis similar to patients with moderate AS in comparison to 'high gradient' severe AS, which suggests that prognosis may be mostly impacted by 'valvular load' rather than 'arterial load'. Therefore, it appears that in patients with severe AS (mean gradient $\geq 40 \mathrm{~mm} \mathrm{Hg}$ ) with preserved EF, reduction in valvular load is the primary determinant of outcome rather than the arterial load imposed by highblood pressure.

After successful AVR, the high-haemodynamic load caused by the stenotic valve is released and Zva is decreased. Thus, the prognosis of those patients after AVR would not be affected by preoperative Zva. Although Zva is an additional measure of global haemodynamic load to the myocardium, this parameter may not directly correlate with the amount of myocardial damage, which is determined not only by the one-time value of Zva (haemodynamic load), but is also determined by the length of the load to which the myocardium is exposed. 
LIMITATIONS

This was a retrospective study performed in a single echocardiography laboratory, and, therefore, variability of the echocardiographic data was minimised. However, the results may not be applicable to other institutions due to differences in healthcare and patient population. Second, Zva is consisted of multiple factors including blood pressure. It is possible that Zva measure may be highly variable according to the systolic blood pressure at the time of echocardiography. Also, the Zva parameter is based on echocardiographic measurements, so participants with fundamental limitations of ultrasonography may have influenced the data. Third, given the retrospective nature of the study, there may have been unmeasured variables that affected the prognosis. Lastly, the sample size may be too small to be able to detect difference in survival of $<18 \%$ between the groups. Therefore, it is possible that a smaller difference in survival between the groups could have been present, but was not detected in our study. Nevertheless, we did not find a major difference between the groups.

\section{CONCLUSION}

Our results indicate that preoperative Zva does not have independent prognostic value in elderly patients with severe AS with preserved EF after AVR. Careful interpretation and application of Zva with other risk parameters are essential. Further larger prospective studies are needed to elucidate these controversial results in order to apply Zva parameter more efficiently in clinical practice.

Acknowledgements The authors thank Gillian Murphy for secretarial help. Preliminary results of this study was presented as an abstract at the 19th World Congress on Heart Disease meeting held in Boston, MA, U.S.A. in 2014.

Contributors MK conceived, designed, participated in data collection, analysis, interpretation and drafting of the manuscript. MQN and PCM participated in data collection, analysis and interpretation and provided intellectual content. MHT participated in data analysis and interpretation, and provided intellectual content. MB and HPC interpreted data and provided intellectual content. All authors approved the final version of the manuscript.

Funding This study was supported by American Heart Association Clinical Research Programme 13 CRP17300021 and A J and Sigismunda Palumbo Charitable Trust.

Competing interests None declared.

Ethics approval Mayo Foundation Institutional Review Board.
Provenance and peer review Not commissioned; externally peer reviewed.

Data sharing statement Our institutional review board policy for this study will not allow data sharing.

Open Access This is an Open Access article distributed in accordance with the Creative Commons Attribution Non Commercial (CC BY-NC 4.0) license, which permits others to distribute, remix, adapt, build upon this work noncommercially, and license their derivative works on different terms, provided the original work is properly cited and the use is non-commercial. See: http:// creativecommons.org/licenses/by-nc/4.0/

\section{REFERENCES}

1. lung $B$, Baron G, Butchart EG, et al. A prospective survey of patients with valvular heart disease in Europe: the Euro Heart Survey on Valvular Heart Disease. Eur Heart J 2003;24:1231-43.

2. Briand M, Dumesnil JG, Kadem L, et al. Reduced systemic arterial compliance impacts significantly on left ventricular afterload and function in aortic stenosis: implications for diagnosis and treatment. J Am Coll Cardiol 2005;46:291-8.

3. Hachicha Z, Dumesnil JG, Bogaty P, et al. Paradoxical low-flow, low-gradient severe aortic stenosis despite preserved ejection fraction is associated with higher afterload and reduced survival. Circulation 2007;115:2856-64.

4. Hachicha Z, Dumesnil JG, Pibarot P. Usefulness of the valvuloarterial impedance to predict adverse outcome in asymptomatic aortic stenosis. J Am Coll Cardiol 2009;54:1003-11.

5. Lancellotti $\mathrm{P}$, Donal $\mathrm{E}$, Magne $\mathrm{J}$, et al. Risk stratification in asymptomatic moderate to severe aortic stenosis: the importance of the valvular, arterial and ventricular interplay. Heart 2010;96:1364-71

6. Rieck AE, Gerdts E, Lonnebakken MT, et al. Global left ventricular load in asymptomatic aortic stenosis: covariates and prognostic implication (the SEAS trial). Cardiovasc Ultrasound 2012;10:43

7. Katsanos S, Yiu KH, Clavel MA, et al. Impact of valvuloarterial impedance on 2-year outcome of patients undergoing transcatheter aortic valve implantation. J Am Soc Echocardiogr 2013;26:691-8.

8. Levy F, Luc Monin J, Rusinaru D, et al. Valvuloarterial impedance does not improve risk stratification in low-ejection fraction, low-gradient aortic stenosis: results from a multicentre study. Eur $J$ Echocardiogr 2011:12:358-63.

9. Lang RM, Bierig M, Devereux RB, et al. Recommendations for chamber quantification: a report from the American Society of Echocardiography's Guidelines and Standards Committee and the Chamber Quantification Writing Group, developed in conjunction with the European Association of Echocardiography, a branch of the European Society of Cardiology. J Am Soc Echocardiogr 2005;18:1440-63.

10. Nagueh SF, Appleton CP, Gillebert TC, et al. Recommendations for the evaluation of left ventricular diastolic function by echocardiography. J Am Soc Echocardiogr 2009;22:107-33.

11. Baumgartner $\mathrm{H}$, Hung J, Bermejo J, et al. Echocardiographic assessment of valve stenosis: EAE/ASE recommendations for clinical practice. J Am Soc Echocardiogr 2009;22:1-23; quiz 101-2.

12. Nishimura RA, Otto CM, Bonow RO, et al. 2014 AHA/ACC Guideline for the Management of Patients With Valvular Heart Disease: a report of the American College of Cardiology/American Heart Association Task Force on Practice Guidelines. Circulation 2014;129:e521-643.

13. Jander $\mathrm{N}$, Minners J, Holme I, et al. Outcome of patients with low-gradient "severe" aortic stenosis and preserved ejection fraction. Circulation 2011;123:887-95. 